\title{
EFECTO DE DISTINTOS ANTIMICROBIANOS SOBRE EL CRECIMIENTO DE LISTERIA MONOCYTOGENES
}

Effect of different antimicrobial agents on the growth of Listeria monocytogenes.

\section{González-Bermúdez C.A.*; Sánchez-Samper E.P.; Padalino M; Frontela-Saseta C.}

Departamento de Tecnología de los Alimentos, Nutrición y Bromatología. Facultad de Veterinaria. Campus Universitario de Espinardo. 30100 Murcia.

*Autor para correspondencia: Carlos Alberto González Bermúdez. Tel.: 0044868884798 , E-mail: Cagb1@um.es

\section{RESUMEN}

En este estudio se ha evaluado la eficacia de dos ácidos orgánicos contemplados en la lista positiva de aditivos alimentarios, el lactato sódico (E-325) y el diacetato sódico (E-262), sobre el crecimiento de Listeria monocytogenes. Estos aditivos se adicionaron en diferentes concentraciones a un medio de cultivo líquido, determinando el incremento de densidad óptica del medio a $600 \mathrm{~nm}$ durante 24 horas a $37{ }^{\circ} \mathrm{C}$, con respecto al medio sin inocular que se tomó como blanco, realizando la medida cada hora. El incremento de absorbancia se midió con respecto al tiempo, evaluando el crecimiento de la bacteria a través de la interpretación de la tasa máxima de incremento de absorbancia $(\mu)$ y el tiempo mínimo requerido para detectar un incremento en la densidad óptica del medio $(\lambda)$. Este último parámetro se puede equiparar al tiempo de latencia o tiempo de adaptación al medio. Así, para el lactato sódico, se observó que ejerce un efecto negativo dosis dependiente sobre el crecimiento de L. monocytogenes, prolongando el tiempo que necesitó la bacteria para adaptarse al medio de cultivo $(\lambda)$, sin afectar a la tasa de crecimiento $(\mu)$ una vez que esta comenzó a crecer. El diacetato sódico mostró ser más efectivo que el lactato sódico frente al crecimiento de la bacteria, incrementando el tiempo de adaptación al medio, así como disminuyendo la tasa de crecimiento. Además, el diacetato sódico consiguió inhibir de forma completa el crecimiento de la bacteria a concentraciones iguales o superiores a $0.2 \%$.

Palabras clave: Diacetato sódico, lactato sódico, Listeria monocytogenes, inhibición, medio de cultivo.

\section{ABSTRACT}

The effectiveness of two organic acids included in the positive list for additives, sodium lactate (E-325) and sodium diacetate (E-262), was evaluated against Listeria monocytogenes growth. Different concentrations of these additives were added to the liquid culture medium. The optical density increments at $600 \mathrm{~nm}$ was 
measured for a 24 hours period under $37^{\circ} \mathrm{C}$, using non-inoculated medium as blank. The measurements were taken every hour in sterile 96 wells plates each. After this analysis, a graphical representation of absorbance increment against time was done, extrapolating the maximum absorbance increment rate $(\mu)$ and the minimum time required to detect an absorbance increment $(\lambda)$ from the graphic. These two parameters made possible to evaluate the bacterial growth. After the analysis of $\lambda$ and $\mu$ for lactate concentrations, a negative effect in bacterial growth was observed, extending $\lambda$ value. Nevertheless, once the bacterial growth started, any effect on $\mu$ value was detected. A higher inhibitory effect was observed after the analysis of these parameters for diacetate concentrations, an extension on $\lambda$ value as well as a $\mu$ value descent was found. In this way, a total inhibition of growth occurred when diacetate concentration was $0,2 \%$ or higher.

Key Words: Sodium lactate, sodium diacetate, Listeria monocytogenes, inhibition, culture medium.

\section{INTRODUCCIÓN}

Listeria monocytogenes es un patógeno emergente, cocobacilar y gram + , que ha cobrado especial importancia como microorganismo responsable de brotes asociados al consumo de alimentos a partir de la última década del siglo XX (Schlech 2000). Este patógeno fue descrito por vez primera por Pirie (1927), como agente causal de una enfermedad que afectaba a roedores de la sabana del sur de África denominada "Tiger River disease", pero no fue hasta los años 1970-1980 cuando se consideró a L. monocytogenes como un serio problema para la salud asociado al consumo de alimentos contaminados, alcanzando en Estados Unidos y Europa niveles de mortalidad entre el $20-40 \%$ en los grupos más susceptibles de la población (niños, ancianos, pacientes inmunodeprimidos y fetos), siendo una tasa excepcionalmente alta para una enfermedad transmitida por alimentos (Schlech 2000). Desde entonces, la notificación de casos de listeriosis alimentaria se ha ido incrementando según los informes anuales de la European Food Safety Authority (EFSA) y del European Center of Disease Control (ECDC), respecto a la incidencia de listeriosis en países de la Unión Europea en base a los casos notificados desde el año 1999 hasta el 2006 (Denny y McLauchlin 2008). Los principales alimentos implicados en estos brotes fueron alimentos Listos para el Consumo (LPC), siendo estos los definidos como "aquellos destinados al consumo directo sin necesidad de ser cocinados o sometidos a otro tipo de transformación capaz. de eliminar o reducir a un nivel aceptable los microorganismos peligrosos", tal y como define el Artículo 2, apartado "g", del Reglamento (CE) $n^{\circ}$ 2073/2005 de la Comisión, de 15 de noviembre de 2005 . La mayoría de casos de listeriosis alimentaria están asociados al consumo de alimentos que no cumplen las normas vigentes relativas a la presencia de L. monocytogenes en alimentos (EFSA, 2007; FAO/OMS, 2004), existiendo una relación directa entre, el nivel de contaminación de un alimento y la presencia de sintomatología en el consumidor. De forma general, la mayoría de alimentos implicados tuvieron un nivel de contaminación superior a 1000 ufc (unidades formadoras de colonias)/g, (Tompkin 2002), por lo que la International Commission on Microbiological Specification for Foods (ICMS 1996), destinó este límite como un nivel de contaminación aceptable, sin entrañar riesgo para la salud de los consumidores.

L. monocytogenes es un microorganismo muy ubicuo, colonizando diferentes ambientes, entre ellos, las plantas de procesado de alimentos, a las que accede principalmente vehiculada por medio de alimentos de origen vegetal y animal crudos, equipos de transporte o por los propios trabajadores, que pueden actuar como portadores sanos (Vogel et al., 2001; Moretro \& Langsrud 2004). La protección del consumidor pasará por prevenir la contaminación de los alimentos por L. monocytogenes (Tompkin 2002). Por lo tanto, la industria alimentaria se enfrenta al hecho de que este microorganismo crece 
en condiciones extremas, en comparación con otros microorganismos contaminantes de alimentos, siendo anaerobia facultativa, halotolerante y capaz de crecer bajo un amplio rango de temperatura, entre 4 y $50{ }^{\circ} \mathrm{C}$ (Rocourt \& Cossart, 1997; Martin \& Fisher, 1999). Además, L. monocytogenes es capaz de formar biofilms y resistir las acciones de limpieza y desinfección rutinarias (Carpentier \& Chassaing 2004). Por todo ello, se hace muy difícil la eliminación de L. monocytogenes de las plantas de procesado de alimentos.

Estas características hacen que los alimentos LPC, adquieran gran importancia en la epidemiología de los brotes de listeriosis alimentaria. La mayoría de estos alimentos van a ser sometidos a tratamientos de esterilización durante su fabricación, no obstante, previamente al envasado son manipulados, entrando en contacto con distintas superficies y/o maquinaria, favoreciendo la contaminación de los mismos. Una vez en el alimento, L. monocytogenes es capaz de multiplicarse hasta alcanzar recuentos microbianos elevados, ya que suele ser resistente a los conservantes o condiciones de conservación habituales en este tipo de alimentos tales como el vacío, la atmósfera modificada o las sales de conservación. Cuando el consumidor consume este tipo de alimentos, no los somete por lo general, a un cocinado que permita reducir la carga microbiana contaminante a unos niveles aceptables, quedando así expuesto al patógeno (Neetoo et al., 2008; Mejlholm \& Dalgaard, 2007; Zhu et al., 2005; Mataragas et al., 2003). Esta situación hace que, dada la dificultad de eliminar la posibilidad de contaminación del alimento durante su procesado, la industria alimentaria y las autoridades sanitarias quieran buscar nuevas alternativas en la conservación de alimentos, eficaces en el control de $L$. monocytogenes. A su vez, debemos considerar el hecho de que, el consumidor actual, demanda alimentos seguros y "naturales", asociando este término a un bajo contenido en conservantes artificiales (McEntire et al., 2003).
Atendiendo a esta necesidad, el objetivo del presente estudio ha sido evaluar el efecto de dos ácidos orgánicos, lactato y diacetato sódico (E325 y E-262), sobre el crecimiento de una cepa de L. monocytogenes en medio de cultivo líquido, como paso previo a su posible empleo como ingrediente en la fabricación de alimentos LPC. La forma no disociada de estos ácidos, va a penetrar en el interior del citoplasma bacteriano, disociándose y liberando protones que acidifican el medio intracelular. A través de una bomba ATP-asa de membrana, la bacteria expulsa estos protones para estabilizar el $\mathrm{pH}$, agotando las reservas energéticas y repercutiendo sobre la tasa de multiplicación bacteriana (Davidson 1996). Ambos ácidos se encuadran dentro de la lista positiva de aditivos alimentarios, considerándose como sustancias GRAS (Generally Recognized as Safe) por la Food and Drug Administration (FDA) de EEUU en el Code of Federal Regulations (CFR, 2010) y acogiéndose al principio quantum satis para su uso dentro de la UE (Directivas 95/2/CE y 2006/52/CE del Parlamento Europeo y del Consejo).

\section{MATERIAL Y MÉTODOS}

\section{Cepa de Listeria monocytogenes y condicio- nes de cultivo}

Para el aislamiento de Listeria monocytogenes se utilizó el método de detección en medio de cultivo RAPID'L mono (Biomereux, Francia), previo enriquecimiento con caldo FRASER (BioRad, America) (NF EN ISO 112902), a partir de $25 \mathrm{~g}$ de alimentos cárnicos LPC (patés a la pimienta y patés de champagne) adquiridos en distintos establecimientos de alimentación de la ciudad de Murcia. A partir de colonias sospechosas se realizó siembra en triple estría sobre placa de Brain Heart Agar (BHA, Merk, Alemania) para obtener cultivos puros, incubando $24 \mathrm{~h}$ a $37{ }^{\circ} \mathrm{C}$. Tras la incubación, se realizó una verificación del género Listeria mediante test catalasa y test oxidasa. 
Solo aquellos cultivos que fueron Catalasa (+) y Oxidasa (-) se sometieron a identificación de especie mediante ensayos enzimáticos y de fermentación de azúcares con galería API® Listeria (Biomereux, Francia). Una vez identificada una cepa pura, se procedió a su incubación en caldo Brain Heart Infusion (BHI, Merk, Alemania) durante 18 horas a $37^{\circ} \mathrm{C}$ y a la creación de un stock en BHI con $20 \%$ de glicerol, manteniéndolo en congelación a $-80{ }^{\circ} \mathrm{C}$. hasta el momento de su utilización.

\section{Efecto antimicrobiano del lactato sódico y diacetato sódico}

Para la realización de los experimentos, se empleó una modificación del medio BHI propuesta por Antwi et al., (2007), añadiendo D(+)-glucosa (Sigma-Aldrich, España) y extracto de levadura (Pronadisa, España). Para los estudios de evaluación del efecto antimicrobiano del lactato sódico (Fluka, Sigma-Aldrich, España), este se adicionó con las concentraciones de $1.5 \%, 2 \%, 2,5 \%$ y $3.5 \%$. En el caso del diacetato sódico (Fluka, Sigma-Aldrich, España), las concentraciones empleadas fueron $0.1 \%, 0.15 \%, 0.2 \%, 0.25 \%$ y $0.3 \%$. Estas concentraciones fueron seleccionadas basándose en estudios de formulación de diferentes alimentos con estos aditivos en los que mostraron un efecto negativo sobre el crecimiento de bacterias Gram +, siendo el principal limitante a su uso las alteraciones sensoriales y el rechazo por los consumidores si se empleaban por encima de estas concentraciones (Maks et al., 2010; Jofré et al., 2008; Marcos et al., 2008; Blom et al., 1997). Las condiciones de incubación de estos medios fueron $37^{\circ} \mathrm{C}$ durante 24 horas, en condiciones de aerobiosis.

\section{Evaluación del crecimiento de Listeria mono- cytogenes}

Para la estimación y evaluación del crecimiento de L. monocytogenes se empleó un mé- todo turbidimétrico, midiendo la variación de la absorbancia del medio líquido, durante 24 horas a $37{ }^{\circ} \mathrm{C}$, a una longitud de onda de $600 \mathrm{~nm}$. La aplicación de las técnicas turbidimétricas al crecimiento microbiano es posible gracias a la relación existente entre la variación de la absorbancia del medio y el número de bacterias en suspensión (Francoise et al., 2005; Dalgaard \& Koutsoumanis, 2001). En este trabajo, se ajustó en primer lugar la carga microbiana inicial del cultivo a concentraciones próximas a $10^{8} \mathrm{ufc} / \mathrm{ml}$ en la escala McFarland, empleando un densitómetro Densimat ${ }^{\circledR}$ (Biomereux, Francia) y posteriormente se realizaron diluciones decimales hasta alcanzar una concentración de $10^{5} \mathrm{ufc} / \mathrm{ml}$.

El estudio se realizó en placas de microtitulación de 96 pocillos estériles, con 8 filas x 12 columnas, (Nunc, Thermo Scientific, USA), basándonos en los estudios de Blom et al., (1997) y Bredholt et al., (1999). La primera columna se utilizó como control positivo para poder determinar el crecimiento bacteriano en el medio sin acción de ninguno de los ácidos orgánicos evaluados. Para medir la absorbancia del medio para cada una de las concentraciones de ácidos orgáncos empleadas, se dejaron seis columnas sin inocular, sirviendo estas como control negativo. La incubación de las placas se realizó durante 24 horas a $37^{\circ} \mathrm{C}$, midiendo la absorbancia cada hora, utilizando para ello un Multilector de Placas Microtiter Synergy HT (Biotek, USA) y un Software de recopilación y tratamiento de datos Gen ${ }^{5}$ (Biotek, USA).

Para cada columna y hora de lectura se calculó un valor de absorbancia medio, obteniendo el incremento de absorbancia ( $\Delta \mathrm{abs})$. Posteriormente se representó gráficamente, para cada una de las condiciones de cultivo, el $\Delta$ abs con respecto al tiempo, obteniendo una curva sigmoidea, con una fase intermedia de incremento exponencial de la absorbancia., que se corresponde a la fase exponencial de crecimiento microbiano (Dalgaard y Kousoumanis, 2001). Los parámetros principales que van a definir el crecimiento microbiano en los métodos 
de recuento en placa son el tiempo de latencia o tiempo que tarda la bacteria en adaptarse al medio y comenzar a crecer y la tasa máxima de crecimiento (Perni et al., 2005). Al trabajar con absorbancias, la equivalencia a estos parámetros las encontramos en la tasa máxima de $\Delta$ abs $(\mu)$ y en el tiempo mínimo requerido para detectar un incremento en la densidad óptica del medio $(\lambda)$. A partir de cada una de las gráficas obtenidas, el parámetro $\mu$ se estimó mediante regresión lineal en la fase de la curva donde la absorbancia presentó un incremento exponencial, siendo este parámetro la pendiente de la recta obtenida. El parámetro $\lambda$ se estimó prolongando esta recta sobre el eje de abcisas, siendo su valor el punto de corte con dicho eje (Zwietering et al., 1990).

\section{Análisis estadístico}

El análisis estadístico se realizó mediante análisis de varianza, ANOVA de un factor, para estudiar el efecto de las distintas concentraciones de cada aditivo sobre $\mu$ y $\lambda$. Con el fin de establecer las diferencias por parejas, entre las medias de cada variable, se realizó un test de Tukey. El nivel de significación estadístico fijado para todos los análisis fue de $p<0.05$. Se utilizó el programa estadístico SPSS versión 15.0 para Windows.

\section{RESULTADOS}

En la Figura 1 se muestra la gráfica obtenida al representar el $\Delta$ abs frente al tiempo para las diferentes concentraciones de lactato. En la Figura 2 se muestra la gráfica obtenida al representar el $\Delta$ abs frente al tiempo para las diferentes concentraciones de diacetato sódico.

Para el caso del lactato sódico los parámetros $\mu$ y $\lambda$ obtenidos se han representado en la Tabla 1. Se puede observar que, tan solo se encontraron diferencias estadísticamente significativas $(\mathrm{P}<0.05)$ en el parámetro $\mu$ del medio al que se le había adicionado una concentración de 2,5\% de lactato sódico, siendo en este caso significativamente menor $(0,0657 \pm 0,0031)$ en comparación con la del medio sin lactato o con

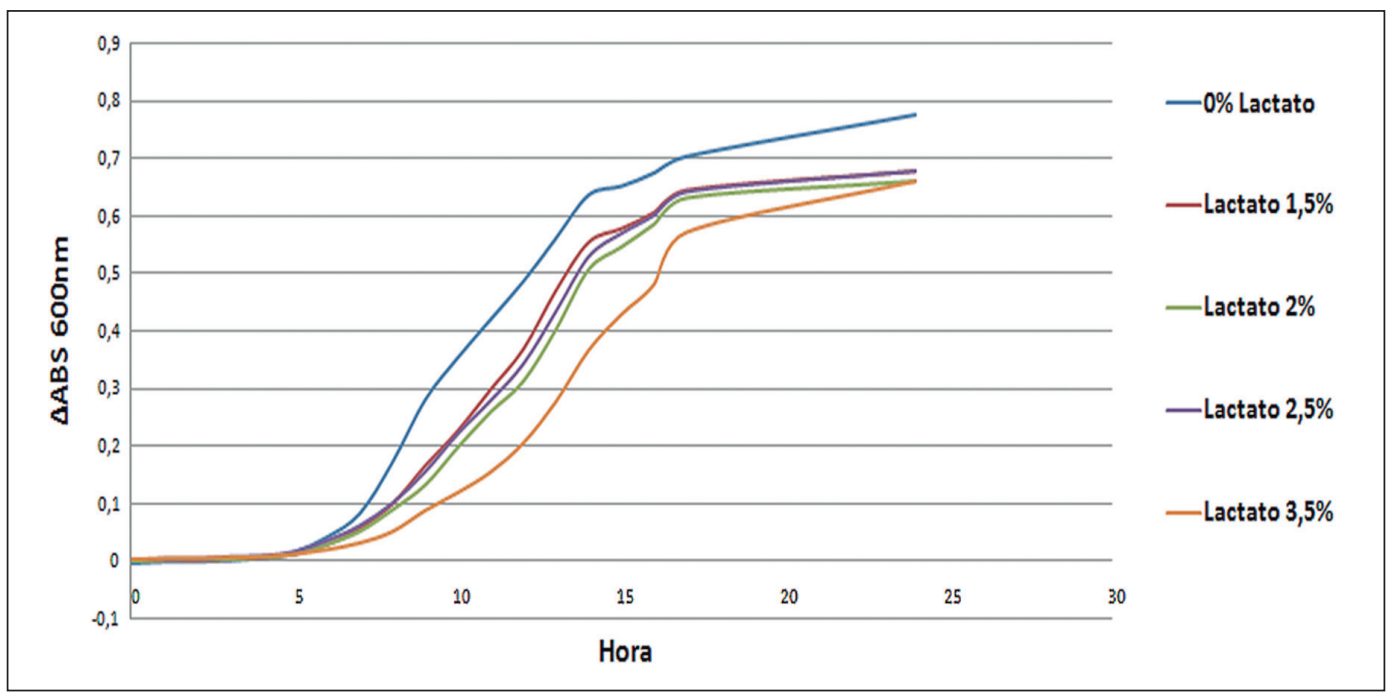

Figura 1. Incremento de la absorbancia, medida a $600 \mathrm{~nm}$, con respecto al tiempo del medio inoculado con Listeria monocytogenes y distintas concentraciones de lactato sódico 


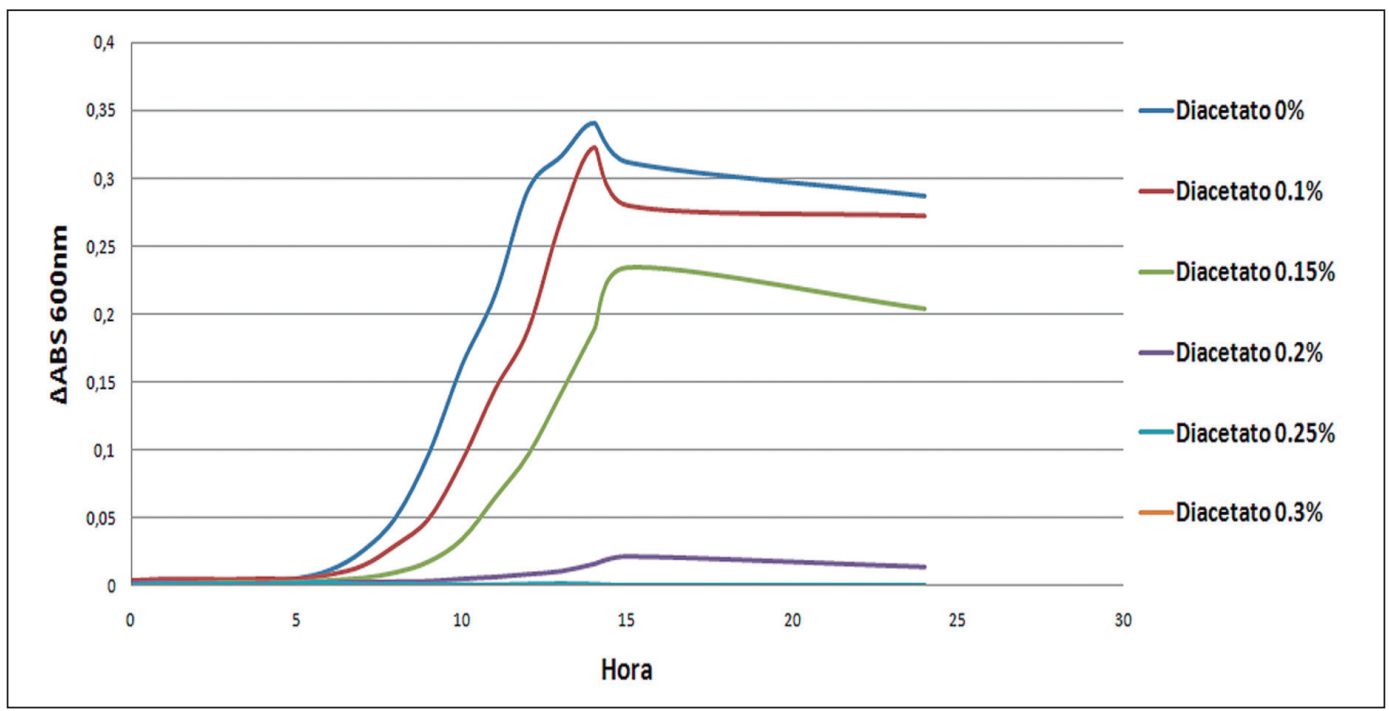

Figura 2. Incremento de la absorbancia, medida a $600 \mathrm{~nm}$, con respecto al tiempo del medio inoculado con Listeria monocytogenes y distintas concentraciones de diacetato sódico

Tabla 1. Valores de los parámetros $\boldsymbol{\mu}$ (tasa máxima de incremento de absorbancia) y $\boldsymbol{\lambda}$ (tiempo mínimo requerido para detectar un incremento en la densidad óptica del medio) para las diferentes concentraciones de lactato sódico empleadas

\begin{tabular}{|c|c|c|}
\hline $\begin{array}{c}\text { Concentración } \\
\text { de aditivo }\end{array}$ & $\boldsymbol{\mu}$ & $\boldsymbol{\lambda}$ \\
\hline $0 \%$ & $0,0746 \pm 0,0045^{\mathrm{a}}$ & $5,454 \pm 0,174^{\mathrm{a}}$ \\
\hline $1,5 \%$ & $0,0722 \pm 0,0046^{\mathrm{ac}}$ & $6,756 \pm 0,128^{\mathrm{b}}$ \\
\hline $2 \%$ & $0,0716 \pm 0,0021^{\mathrm{a}}$ & $6,961 \pm 0,188^{\mathrm{b}}$ \\
\hline $2,5 \%$ & $0,0657 \pm 0,0031^{\mathrm{bc}}$ & $6,269 \pm 0,035^{\mathrm{c}}$ \\
\hline $3,5 \%$ & $0,0745 \pm 0,0034^{\mathrm{a}}$ & $9,094 \pm 0,197^{\mathrm{d}}$ \\
\hline
\end{tabular}

(a-d) Diferentes letras como superíndice indican diferencias estadísticamente significativas $(\mathrm{p}<0,05)$ dentro de la misma columna entre cada una de las concentraciones de lactato sódico empleadas $(0 \%-3,5 \%)$.

la de los medios que poseían otras concentraciones ensayadas de este aditivo. En cuanto al parámetro $\lambda$, se encontraron diferencias estadísticamente significativas entre el medio $\sin$ lactato y el medio con cualquiera de las concentraciones estudiadas de lactato sódico. Se observó que en el medio control, $\lambda$ fue menor que en el medio al que se le añadió este conser- vante, aumentando de forma significativa al ir incrementando la concentración de lactato sódico hasta alcanzar un valor de $(9,094 \pm 0,197$ horas) para el medio con una concentración de lactato de 3,5\%.

Los parámetros $\mu$ y $\lambda$ para el diacetato sódico son los representados en la Tabla 2. Se encontraron diferencias estadísticamente signi- 
Tabla 2. Valores de los parámetros $\boldsymbol{\mu}$ (tasa máxima de incremento de absorbancia) y $\lambda$ (tiempo mínimo requerido para detectar un incremento en la densidad óptica del medio) para las diferentes concentraciones de diacetato sódico empleadas

\begin{tabular}{|c|c|c|}
\hline $\begin{array}{c}\text { Concentración } \\
\text { de aditivo }\end{array}$ & $\boldsymbol{\mu}$ & $\boldsymbol{\lambda}$ \\
\hline $0 \%$ & $0,0358 \pm 0,0045^{\mathrm{a}}$ & $5,454 \pm 0,174^{\mathrm{a}}$ \\
\hline $0,1 \%$ & $0,0594 \pm 0,0046^{\mathrm{b}}$ & $6,756 \pm 0,128^{\mathrm{b}}$ \\
\hline $0,15 \%$ & $0,037 \pm 0,0036^{\mathrm{c}}$ & $9,531 \pm 0,139^{\mathrm{c}}$ \\
\hline $0,2 \%$ & N.D. & $\infty$ \\
\hline $0,25 \%$ & N.D. & $\infty$ \\
\hline $0,3 \%$ & N.D. & $\infty$ \\
\hline
\end{tabular}

(a-c) Diferentes letras como superíndice indican diferencias estadísticamente significativas $(\mathrm{p}<0,05)$ dentro de la misma columna entre cada una de las concentraciones de diacetato sódico empleadas $(0 \%-0,3 \%)$.

ficativas $(\mathrm{p}<0,05)$ en el valor de $\mu$ para todos los niveles de diacetato evaluados. En concreto se observó que, a medida que aumenta la concentración de diacetato sódico, el valor de este parámetro disminuyó, llegando a alcanzar valores que pueden considerarse despreciables a partir de una concentración de $0,2 \%$ de diacetato sódico. En cuanto al parámetro $\lambda$, también se encontraron diferencias estadísticamente significativas para todos los niveles de diacetato, aumentando de forma significativa conforme aumenta la concentración del aditivo. A partir de una concentración de $0,2 \%$ de diacetato sódico consideramos que la fase de latencia se prolonga durante todo el tiempo del ensayo $(\infty)$, al no detectarse crecimiento.

\section{DISCUSIÓN}

En el presente estudio se ha observado que, a medida que se incrementa la concentración de lactato sódico en el medio de cultivo a $37^{\circ} \mathrm{C}$ en aerobiosis, se ejerce un efecto negativo sobre el crecimiento de L. monocytogenes, encontrando la máxima inhibición de dicha bacteria a una concentración de lactato sódico de 3,5\%. Es- tos resultados coinciden con los obtenidos por Blom et al. (1997), Jofré et al., (2008), Marcos et al., (2008) y Maks et al., (2010) en estudios realizados sobre la adición de este aditivo en distintos alimentos. En concreto se observó que, el lactato no modificó de forma significativa el valor $\mu$, obteniendose curvas de incremento de absorbancia con fases de incremento exponenciales de trayectorias paralelas. El mayor efecto se observó sobre $\lambda$, el cual se incrementó de forma significativa a medida que aumentó la concentración de lactato, pasando de un valor inicial aproximado de $5 \mathrm{~h}$ en el control, a un valor aproximado de $9 \mathrm{~h}$ con la máxima concentración del aditivo $(3,5 \%)$. Esto se traduce en que el lactato alarga el tiempo que necesita L. monocytogenes para adaptarse al medio de cultivo aunque una vez que se ha adaptado no afecta de forma negativa a la tasa de incremento de la absorbancia en la fase exponencial.

Por otro lado, según los resultados obtenidos en nuestro estudio, el diacetato presenta un efecto inhibitorio sobre el crecimiento de $L$. monocytogenes, confirmando estos resultados otros estudios previos (Schlyter et al., 1993; Blom et al., 1997; Marcos 2007). Así se encon- 
tró que este efecto inhibitorio fué mayor conforme aumentó la concentración de diacetato, disminuyendo de forma significativa el valor de $\mu$ y aumentando el valor de $\lambda$, lo que se traduce en un incremento en el tiempo de adaptación de la bacteria al medio de cultivo y una menor tasa de crecimiento de la misma. En concreto, bajo las condiciones del presente estudio, el diacetato inhibió totalmente el crecimiento de L. monocytogenes a partir de una concentración del $0,2 \%$.

Con todo ello, se puede concluir que, el lactato y diacetato sódico son antimicrobianos naturales capaces de inhibir de forma parcial o completa, en función de la concentración empleada, el crecimiento de L. monocytogenes. Debido a su inocuidad para el consumidor y a la ausencia de límites legales de uso, se hace necesaria la evaluación de su empleo en la fabricación de alimentos listos para el consumo.

\section{AGRADECIMIENTOS}

Agradecemos a los profesores Carmen Martínez Graciá y Gaspar F. Ros Berruezo la ayuda, supervisión y confianza depositada y, a nuestra compañera Patricia Peso Echarri por la colaboración en este trabajo de investigación.

\section{BIBLIOGRAFÍA}

ANTWI M., BERNAERTS K., VAN IMPE J.F., GEERAERD A.H., 2007. Modelling the combined effects of structured food model system and lactic acid on Listeria innocua and Lactococcus lactis growth in mono- and coculture. Int. J. Food Microbiol. 120: 7184.

BLOM H., NEBRINK E., DAINLY R., HAGTVEDT T., BORCH E., NIESSEN H., NESBAKKEN T. 1997. Addition of $2.5 \%$ lactate and $0.25 \%$ diacetate controls growth of Listeria monocytogenes in vacuum-packed servelat sausage and cooked ham stored at $4^{\circ} \mathrm{C}$. Int. J. Food Microbiol. 38: 71-76.
BREDHOLT S., NESBAKKEN T., HOLCK A., 1999. Protective cultures inhibit growth of Listeria monocytogenes and Escherichia coli $\mathrm{O} 157: \mathrm{H7}$ in cooked, sliced, vacuumand gaspackaged meat. Int. J. Food Microbiol. 53: 43-52.

CARPENTIER B., CHASSAING D. 2004. Interactions in biofilms between Listeria monocytogenes and resident microorganisms from food industry premises. Int. J. Food Microbiol. 97: 111-122.

CODE OF FEDERAL REGULATIONS (CFR) 2010. Cita:21CFR184,21(3). Disponible en: http://www.accessdata.fda.gov/scripts/cdrh/ cfdocs/cfcfr/CFRSearch.cfm?fr=184.1754

DALGAARD P. \& KOUTSOUMANIS K., 2001. Comparasion of maximum specific growth rates and lag time estimated from absorbance and viable count data by different mathematical models. J. Microbiol. Methods. 43: 183-196.

DAVIDSON P.M., 1996. Conservantes químicos y compuestos antimicrobianos. En: Microbiología de los alimentos fundamentos y fronteras, pp 544-548. Ed. Doyle, Bechaut, Montville. Acribia. 816 pp.

DENNY J. \& MCLAUCHLIN J., 2008. Human Listeria monocytogenes infections in Europe - an opportunity for improved European surveillance. Euro Surveill. 13 (1-3): 1-5.

DIRECTIVA 95/2/CE DEL PARLAMENTO EUROPEO Y DEL CONSEJO de 20 de febrero de 1995 relativa a aditivos alimentarios distintos de los colorantes y edulcorantes. DO no L 61 de 18.3 de 1995.

DIRECTIVA 2006/52/CE DEL PARLAMENTO EUROPEO Y DEL CONSEJO de 5 de julio de 2006 por la que se modifica la Directiva 95/2/CE relativa a aditivos alimentarios distintos de los colorantes y edulcorantes y la Directiva 94/35/CE relativa a los edulcorantes utilizados en los productos alimenticios. DO L 204 de 26 del 7 del 2006.

EFSA 2007. European Food Safety Authority. Scientific Opinion of the Panel on Biologi- 
cal Hazards on a request from the European Commission on Request for updating the former SCVPH opinion on Listeria monocytogenes risk related to ready-to-eat foods and scientific advice on different levels of Listeria monocytogenes in ready to- eat foods and the related risk for human illness. EFSA J, 599: 1-42.

FAO/OMS 2004. Risk assessment of Listeria monocytogenes in ready-to-eat-foods. FAO/ OMS, Roma y Ginebra. Disponible en: http:/www.who.int/foodsafety/publications/ micro/mra_listeria/en/index.html

FRANCOIS K., DEVLIEGHERE F., SMET K., STANDAERT A.R., GEERAERD A.H., VAN IMPE J.F., DEBEVERE J. 2005. Modelling the individual cell lag phase: effect of temperature and $\mathrm{pH}$ on the individual cell lag distribution of Listeria monocytogenes. Int. J. Food Microbiol. 100: 41-53.

INTERNATIONAL COMMISSION ON MICROBIOLOGICAL SPECIFICATION FOR FOODS. 1996. Establishment of sampling plans for microbiological safety criteria for foods in international trade, including recommendations for control of Listeria monocytogenes, Salmonella enteritidis, Campylobacter and enterohemorrhagic E. coli. Codex Committee on Food Hygiene, 29th session, 21 to 25 October 1996. Agenda item 11, CX/ FH 96/91-16. Codex Alimentarius Commission, Rome, Italy.

JOFRÉ A., GARRIGA M., AYMERICH T., 2008. Inhibition of Salmonella sp., Listeria monocytogenes and Staphylococcus aureus in cooked ham by combining antimicrobials, high hydrostatic pressure and refrigeration. Meat Sci. 78: 53-59.

MAKS N., ZHU L., JUNEJA V.K., RAVISHANKAR 2010. Sodium lactate, sodium diacetate and pediocin: Effects and interactions on the thermal inactivation of Listeria monocytogenes on bologna. Food Microbiol. 27: 64-69.

MARCOS M.B. (2007). Mejora de la seguridad alimentaria en productos cárnicos listos para el consumo mediante la aplicación combinada de tecnologías de conservación emergentes. Tesis Doctoral. Universidad de Gerona.

MARCOS M.B., JOFRÉ A., AYMERICH T., MONFORT J.M., GARRIGA M., 2008. Combined effect of natural antimicrobials and high pressure processing to prevent Listeria monocytogenes growth after a chain break during storage of cooked ham. Food Control. 19: 76-81.

McENTIRE J.C., MONTVILLE T.J. AND CHIKINDAS M.L. 2003. Synergy between nisin and select lactates against Listeria monocytogenes is due to the metal cations. J. Food Prot. 66: 1631-1636.

MARTIN, S. E., Y FISHER, C. W., 2000. Listeria monocytogenes. En: Encyclopedia of Food Microbiology vol. 2, pp. 1134-1163. Ed. Robinson R.K., Batt C.A., Patel P.D., Academic Press, London. 2372 pp.

MATARAGAS M., DROSINOS E.H., METAXOPOULOS J., 2003. Antagonistic activity of lactic acid bacteria against Listeria monocytogenes in sliced cooked cured pork shoulder stored under vacuum or modified atmosphere at $4 \pm 2^{\circ} \mathrm{C}$. Food Microbiol. 20: 259-265.

MEJLHOLM, O., DALGAARD, P., 2007. Modeling and predicting the growth boundary of Listeria monocytogenes in lightly preserved seafood. J. Food Prot. 70: 70-84.

MORETRO T \& LANGSRUD S. 2004. Listeria monocytogenes: biofilm formation and persistence in food-processing environments. Biofilms. 1: 107-121.

NEETOO, H., YE, M., CHEN, H., JOERGER, R.D., HICKS, D.T., HOOVER, D.G., 2008. Use of nisin-coated plastic films to control Listeria monocytogenes on vacuum-packaged cold-smoked salmon. Int. J. Food Microbiol. 122: 8-15.

PERNI S., ANDREW P.W., SHAMA G. 2005. Estimating the maximum growth rate from microbial growth curves: definition is everything. Food Microbiol. 22(6): 491-495. 
PIRIE JHH.1927 A new disease of veld rodents, "Tiger River disease." Publ. S. Afr. Inst. Med. Res, 3: 163-186.

REGLAMENTO (CE) No 2073/2005 DE LA COMISIÓN de 15 de noviembre de 2005 relativo a los criterios microbiológicos aplicables a los productos alimenticios. DO L 338 de 22.12.2005: 1-33.

ROCOURT J. \& COSSART P., 1997. Food Microbiology.: fundamentals and frontiers. American Society for Microbiology. Washington D. C., Estados Unidos, 425 pp.

SCHLECH $3^{\text {rd }}$ W.F. 2000. Foodborne Listeriosis. Clin Infect Dis 31: 770 - 775.

SCHLYTER J.H., DEGNAN A.J., LOEFFELHOLZ J.M., GLASS K.A., LUCHANSKY J.B., 1993. Evaluation of sodium diacetate and ALTA 2341 on viabiality of Listeria monocytogenes in turkey slurries. J. Food Prot. 56: 808-810.
TOMPKIN RB. 2002. Control of Listeria monocytogenes in the Food-Processing Environment. J. Food Prot. 65(4): 709-725.

VOGEL BF, HUSS HH, OJENIYI B, AHRENS P, GRAM L. 2001. Elucidation of Listeria monocytogenes Contamination Routes in Cold-Smoked Salmon Processing Plants Detected by DNA-Based Typing Methods. Appli. Environ. Microb., 67(6): 2586 2595.

ZHU M., DU M., CORDRAY J., UK AHN D. 2005. Control of Listeria monocytogenes contamination in ready-to-eat meat products. Compr. Rev. food sci. food safety, 4: 34-42.

ZWIETERING, M.H., JONGENBURGER, I., ROMBOUTS, F.M., VAN'T REIT, K.. 1990. Modeling of the bacterial growth curve. Appl. Environ. Microbio. 56: 1875-1881. 